\title{
Role of the SOX18 protein in neoplastic processes (Review)
}

\author{
MATEUSZ OLBROMSKI ${ }^{1}$, MARZENNA PODHORSKA-OKOŁÓW ${ }^{1}$ and PIOTR DZIĘGIEL ${ }^{1,2}$ \\ ${ }^{1}$ Department of Histology and Embryology, Wroclaw Medical University, 50-368 Wroclaw; \\ ${ }^{2}$ Department of Physiotherapy, University School of Physical Education, 51-617 Wroclaw, Poland
}

Received January 8, 2018; Accepted May 2, 2018

DOI: $10.3892 / \mathrm{ol} .2018 .8819$

\begin{abstract}
There is a high demand for anticancer drugs due to the fact that the chemotherapeutics currently used have numerous side effects, which lowers the patient's quality of life. However, the latest antibody therapies are extremely expensive, hence the requirement to identify novel, equally effective but low-toxic treatments that have limited side effects. As a result of this, a number of research centres around the world are attempting to identify novel molecular markers that could be effective targets for anticancer therapy in the future. The SOX18 protein has been suggested to be a significant diagnostic and prognostic marker in various types of cancer. SRY-related HMG-box 18 (SOX18) is an important transcription factor involved in the development of cardiovascular and lymphatic vessels during embryonic development. In addition, it is involved in the progression of atherosclerosis and wound-healing processes. It has been observed that its level is higher in a number of cancer types, including melanoma, pancreas, stomach, liver, breast, lung, ovarian and cervical cancer. Furthermore, an association between a high expression of SOX18 in gastric cancer stromal cells and a poor prognosis has been demonstrated. The literature indicates how complex the pathogenesis of cancer is. Knowing the molecular basis of the pathogenesis of the tumor will allow for the effective use of targeted therapy, which may result in a higher success in treating patients. It is therefore important to identify novel and effective therapies as well as new proteins that could be potential markers. The SOX18 family, represented by the SOX18 protein, seems to be in this respect a promising element in modern anticancer therapy.
\end{abstract}

\section{Contents}

1. Introduction

2. SOX protein family

Correspondence to: Professor Piotr Dzięgiel, Department of Histology and Embryology, Wroclaw Medical University, Chalubinskiego 6a, 50-368 Wroclaw, Poland

E-mail: piotr.dziegiel@umed.wroc.pl

Key words: SOX18, malignant tumors
3. Group F of SOX proteins

4. SOX18 protein

5. Breast cancer

6. Ovarian and cervical cancer

7. Stomach and liver cancer

8. Non-small cell lung cancer

9. Skin lymphomas

10. Osteosarcoma

11. Conclusions

\section{Introduction}

The term cancer refers to a group of diseases during the course of which an increase in the number of cells with altered genotype and phenotype is observed. The characteristic feature of cancer cells is primarily their uncontrolled and unrestrained proliferation, as well as their autonomy, which underlies the carcinogenesis process. Due to the many changes on a molecular basis (mutation among others), an increase in cancer aggressiveness is a very complex and long-lasting process that usually proceeds asymptomatically. A changed sensitivity to a number of growth factors (cytokines, chemokines and others) is responsible for the unlimited number of cell divisions, the apoptosis impairment, the uncontrolled proliferation and the masking and defence against the host's immune system. One of the final stages in cancer progression is the formation of cardiovascular and lymphatic vessels (angio- and lymphangiogenesis, respectively), thanks to which the tumor grows in volume and acquires the ability to metastasize.

Due to the complexity of the cancer progression process and, in the case of many types or cancer, the lack of effective therapy regimens, new molecular markers which could be potential targets for anti-cancer therapy are increasingly sought after. The proteins of the SRY-related HMG-box (SOX) family seem to be a promising element in cancer therapy in this aspect (1). The family of SOX proteins was isolated and selected in 1990 on the basis of the presence of the high mobility group box (HMG) conservative protein domain, initially characteristic for the sex-determining factor found on the Y chromosome [SRY (sex determining region $Y)]$ (1). Typical for SOX family proteins, the HMG domain (which consists of 79 amino acids) is characterized by a $50 \%$ homology to that occurring in the SRY gene. The SOX proteins, which are transcription factors, bind to the 5'-(A/T)(A/T)CAA(A/T)G-3' sequence precisely through the HMG domain, thus allowing other transcription 
factors to bind (Fig. 1) (2,3). The SOX family is comprised of approximately 20 proteins, divided into 8 main groups (from A to $\mathrm{H}$ ) due to their homology and the functions they perform in the body $(4,5)$. The F group includes the SOX7, SOX17 and SOX18 proteins, which are involved in the same pathways as the vascular endothelial growth factor (VEGF) (6-9).

The SOX18 protein is one of the most important transcription factors involved in the development of blood and lymphatic vessels during the embryogenesis process. Likewise, studies in humans have shown that mutations in the SOX18 gene may result in serious developmental disorders. SOX18 mutation is responsible for the emergence of the hypotrichosislymphedematelangiectasia (HLT) syndrome $(10,11)$. This disease is characterized by the simultaneous appearance of abnormalities in hair development, the presence of lymphedema and the widening of small blood vessels. Recent research has also shown its participation in the pathogenesis of many cancers (12-15). Since different levels of this protein have been observed in different types of tumors, it seems that it can perform various functions in cancer cells (16).

\section{SOX protein family}

The SRY protein, coded by the $S R Y$ gene-located in the Y chromosome and conditioning sex (17), belongs to the group A of SOX proteins. Group B, which is expressed primarily in the central nervous system, is divided into two subgroups: B1, which consists mainly of activators (SOX1, SOX2 and SOX3), and B2, which contains mainly suppressors of the transcription process (SOX4 and SOX21). Representatives of group $\mathrm{C}$ are the proteins SOX11 and SOX12, whose function has not yet been fully investigated and described. However, an increase in the expression level of the genes SOX4 and SOX21 in non-small cell lung carcinomas has been demonstrated $(18,19)$. The proteins SOX5, SOX6 and SOX13, which belong to group D, are involved in the processes of spermatogenesis and chondrogenesis $(5,20)$. SOX8, SOX9 and SOX10, representatives of group E, are involved in the development of the nervous system and the testicular nuclei (21-23). Group F includes the previously mentioned SOX7, SOX17 and SOX18 proteins. The SOX15 protein is the only representative of group G, and it is involved in the development of the testis, the placenta and the muscular system during the process of embryogenesis (24). The last group of transcription factors from the SOX family is the group H, a representative of which is the SOX30 protein, involved mainly in the process of spermatogenesis (25). Additionally, it has recently been showed that SOX30 has a suppressor function in adenocarcinoma (26).

\section{Group F of SOX proteins}

The representatives of the group F of SOX proteins are the proteins SOX7, SOX17 and SOX18, jointly involved in the development of the cardiovascular and lymphatic systems during the embryogenesis process $(27,28)$. Since the main characteristic of these proteins is their high homology, they are able to take over each other's functions, as demonstrated in a mouse model where the inhibition of the SOX18 gene expression resulted in an increased expression of the SOX7 and SOX17 gene (29).

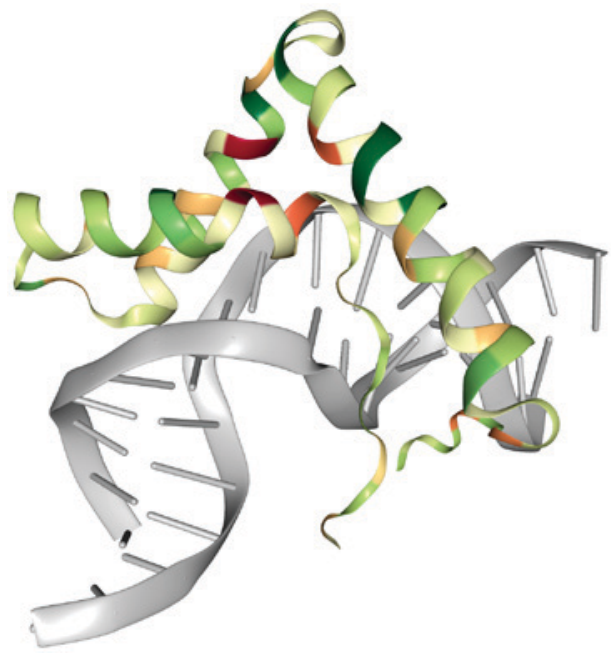

Figure 1. Protein structure and DNA-binding of SOX18. SOX18, SRY-related HMG-box 18 (58).

It has also been proven that, during the embryonic development, SOX18 interacts with the myocyte enhancer factor-2C (MEF2C) transcription factor in the vascular endothelial cells. It has been demonstrated in vivo in a mouse model that the simultaneous silencing of the SOX 18 and MEF2C genes results in serious cardiovascular malformations, which clearly confirms the key role of these transcription factors in the initial stages of the development of blood vessels (30).

An important characteristic of the SOX7, SOX17 and SOX18 proteins is the presence of the amino acid sequence DXXEFD/EQYL, responsible for the interaction with the $\beta$-catenin in the $\mathrm{WNT} / \beta$-catenin pathway $(30,31)$. This pathway is involved in several key physiological processes at every stage of the development of the body, and its disorders lead to the initiation of the carcinogenesis process. Sinner et al have shown that SOX17 acts as an antagonist to the WNT/ $\beta$-catenin pathway by being able to bind simultaneously with the $\beta$-catenin and the t-cell factor (TCF) protein. In vitro studies performed on the SW480 colorectal cancer line model demonstrated that the overexpression of the SOX17 gene inhibits the activity of the $\beta$-catenin/TCF complex, thereby reducing the level of proliferation of the cells of this cancer (32-34).

In turn, in the case of the SOX7 protein, it has been shown that the downregulation of the expression of its gene resulted in the inhibition of the WNT/ $\beta$-catenin pathway in endometrial cancer cells, which correlated positively with a higher malignancy degree of the tumor (33-35).

The role of the proteins of the SOX family, including proteins from the SOX F group, in the development of cancer has been the focus of many research teams around the world for many years. It is worth noting that, despite the high homology of the SOX7, SOX17 and SOX18 proteins, they can act both as stimulators and inhibitors in the carcinogenesis process (17).

\section{SOX18 protein}

In humans, the gene encoding the SOX18 protein is found in the long arm of chromosome 20 in the locus 20q13.3, and it consists of 8,901 base pairs. The product of the SOX18 gene is 

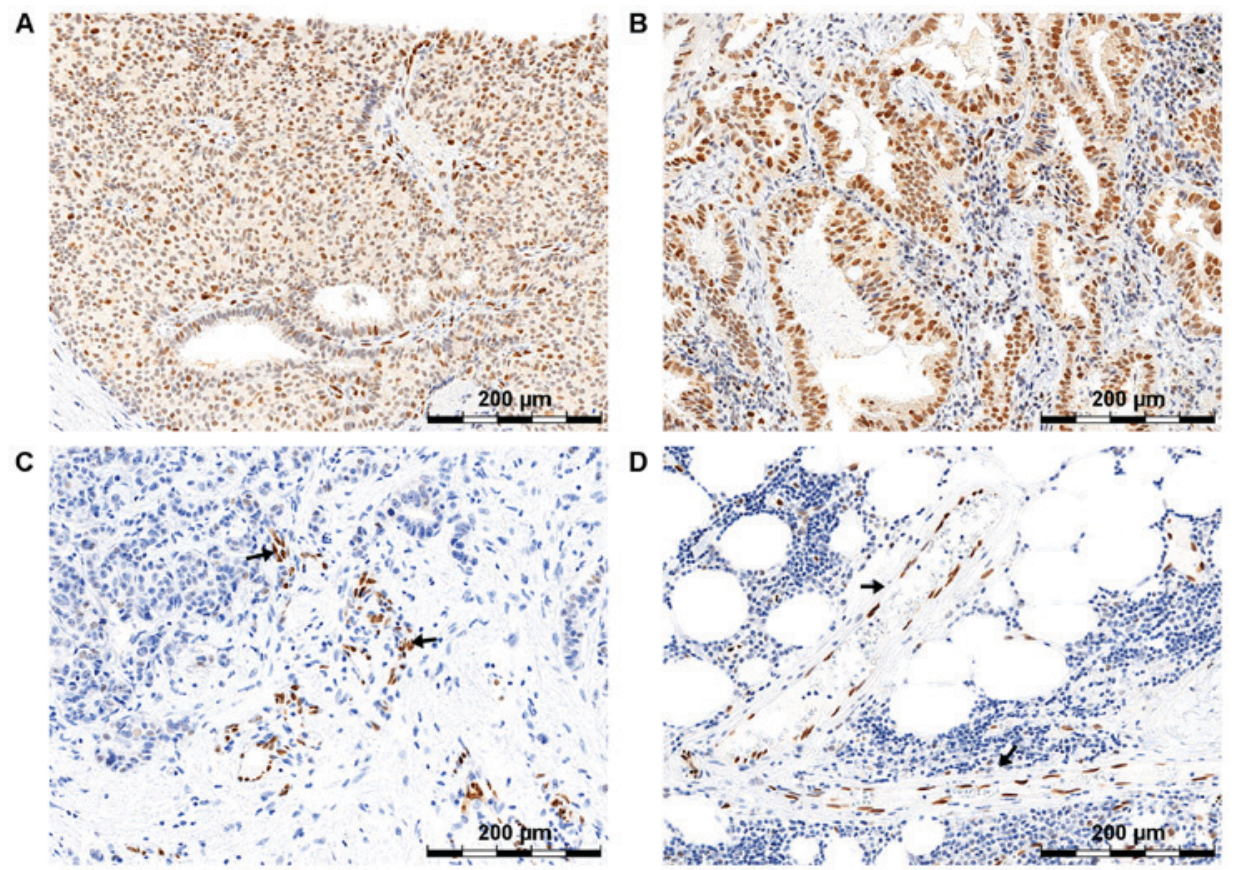

Figure 2. Differentiated expression of SOX18 protein in invasive ductal breast carcinoma and non-small cell lung carcinoma (14). (A and B) Samples presented nuclear expression of SOX18 protein. (C and D) SOX18 expression was also noted in the nuclei of endothelial cells of vessels (indicated with arrows). Original magnification, x600. SOX18, SRY-related HMG-box 18.

a protein made of 384 amino acids with a mass of $41 \mathrm{kDa}(8)$. In the embryonic development, this protein is responsible for the activation of the Prospero Homeobox 1 (Prox-1) promoter and, thus, it is involved in the formation of new blood and lymphatic vessels (28). The initiation of the expression of the SOX18 gene and, therefore, of PROX1, in the early stages of embryogenesis, results in changes in the phenotype of endothelial cells, which causes their migration and fusion (which leads to the formation of a network of lymphatic vessels, the protoplasts of the mature lymphatic system) (28). Among other discoveries, it has been found that in mouse embryos with a silenced SOX18 gene expression, the lymphangiogenesis process is inhibited (28).

In addition to the involvement of the SOX18 protein in the formation of the cardiovascular system in the embryonic development, its presence has also been demonstrated in many cells and tissues of the mature human body, for example in the heart, the lungs, the skeletal muscles, the stomach and the intestines $(8,36)$. Moreover, an increase in the level of this protein has been observed in pathological states such as coronary artery atherosclerosis and would healing $(37,38)$.

Additionally, it has been demonstrated that the SOX18 transcription factor is able to form not only functional homodimeric complexes, but also heterodimeric ones with MEF2C. Furthermore, SOX7 is also capable of creating heterodimers with the Recombining Binding Protein Suppressor of Hairless (RBPJ) protein, and SOX17 with Octamer-binding transcription factor 4 (OCT4) (39). The formation of these complexes allows the transcription factors of the SOX family to bind to distant DNA regions, thereby activating or repressing a larger gene pool.

The observed variable levels of SOX18 in different kinds of cancer (stomach, colorectal, ovarian, endometrium or liver cancer) indicate the role of this transcription factor in tumor progression, metastasis and the development of blood and lymphatic vessels within the tumor.

\section{Breast cancer}

The results of the tests by Pula et al, conducted using the immunohistochemical (IHC) technique on clinical material from 122 cases of Invasive Ductal Breast Carcinoma (IDC), indicated the existence of a significant positive correlation between an increase in the SOX18 protein level and an increase in the tumor malignancy grade (Fig. 2) (14). The expression of the SOX18 factor in the nuclei of the vascular endothelial cells of the tumor was confirmed, suggesting the participation of SOX18 in the angiogenesis process. Interestingly, the results of the in vitro tests on breast cancer human cell lines with increasing aggressiveness (MCF-7, BT-474, SK-BR-3, MDA-MB-231 i MDA-MB-231/BO2), on normal human dermal fibroblasts (MHDF) and on the normal human breast cell line HME1-hTERT confirmed different levels of the SOX18 protein in the breast cancer cell lines and a lack of SOX18 expression in the NHDF and the HME1-hTERT control lines. By using the laser microdissection technique, the authors clearly demonstrated the existence of an increased expression of the SOX18 gene and the SOX7 and SOX17 genes in the vascular endothelial cells, thus confirming the role of these proteins in the angiogenesis process. Additionally, the level of SOX18 mRNA correlated with the mRNA level of the gene $V E G F D$, which indicates the role of this protein in the lymphangiogenesis process. Moreover, it was noted that the breast cancer cell lines not expressing the estrogen receptor (ER-), the progesterone receptor (PR-) and the human epidermal growth factor receptor type 2 (HER2-), i.e., the triple negative, were characterized by a negligible expression of SOX18 (14). This may indicate the involvement of this 
transcription factor in the $\mathrm{Wnt} / \beta$-catenin pathway, responsible for IDC progression, since similar observations made by other research teams had already indicated the contribution of the SOX18 protein to the regulation of TCF transcription factors (33).

In vitro studies by Young et al showed that the inhibition of the expression of the SOX18 transcription factor in the MCF-7 line results in a decrease in the capacity of these cells to migrate because of the destabilization of the actin cytoskeleton (40). As a consequence, it was claimed that SOX18 inhibition caused a significant reduction in the size of induced tumors, as well as a decrease in blood vessel density within the tumor. These observations confirmed the participation of the SOX18 protein in the angiogenesis process. Since the number of blood vessels in the tumor closely correlates with the risk of metastases and shorter survival times, silencing the expression of the SOX18 gene (which leads to the reduction of the blood vessel network within the tumor) may contribute to sensitizing cancer cells to chemotherapeutic agents by reducing the supply of nutrients supplied along with blood through the blood vessels. Additionally, an increase in the level of the SOX18 expression in the MCF-7 line resulted in an increased proliferation and the creation of capillaries from the Human Umbilical Vein Endothelial Cells (HUVEC), ultimately confirming the contribution of the SOX18 protein to the progression of invasive breast cancer (40).

\section{Ovarian and cervical cancer}

Due to the fact that SOX18 is expressed not only by vascular endothelial cells but also by tumor cells themselves, Petrovic et al decided to investigate the role of this transcription factor in cervix cancers (41). So far, the involvement of the SOX18 protein has been confirmed in the Hedgehog pathway in non-cancerous human cells, primarily during embryonic development. Since the Hedgehog pathway remains mainly inactive in the mature cells of the body and its activation is observed in tumors (including cervix cancer), the modulation of the inhibitors of this pathway may be important in future targeted therapy. Petrovic et al demonstrated that the SOX18 protein is regulated by the GL1 and GL2 (glioma-associated oncogene-1, -2) transcription factors, which are modulators of the Hedgehog pathway (39). It was proved that the inhibitors GL1 and GL2, involved in the regulation of the aforementioned pathway, are also responsible for the reduction of the expression level of the SOX18 gene. Moreover, the in vitro tests on the human cervix cancer cell lines HeLa, SiHa and CaSki showed that a SOX18 overexpression did not increase the proliferative potential, but significantly increased the invasiveness and migration of the cells of this cancer (41). This could suggest a contribution of the SOX18 protein to the promotion of tumor progression in cervix cancers.

Other studies carried out on cervix cancer postoperative material were designed to demonstrate the potential of the SOX18 protein as a predictive marker in the chemotherapy of this type of cancer. Pula et al showed that high levels of SOX18 in ovarian cancer cell nuclei significantly correlated with a shorter disease-specific survival (DSS) and a shorter disease-free survival (DFS) (15). Additionally, the in vitro tests on the human ovarian cancer cell lines SK-OV-3 and OVCAR3 and the ovarian normal surface epithelial cell line IOSE 364 indicated the possibility of the participation of the SOX18 protein in the mechanism of tumor cell resistance to chemotherapeutics, since the SK-OV-3 and OVCAR3 cells, characterized by a high drug resistance (including cisplatin), showed high levels of the SOX18 protein.

\section{Stomach and liver cancer}

Research by Jia et al (42) was carried out on material from 196 cases of stomach cancer and the 6 human stomach cancer cell lines SGC7901, MGC803, BGC823, AGS, N87 and MKN45. In these experiments, the authors determined the level of methylation of the naked cuticle homolog (NKD) -1 and -2 gene promoters, SOX18 direct inhibitors, on the aforementioned material. It turned out that the methylation of the NKDl gene is influenced, among others, by the ability of the cancer cells to migrate and create metastases (42). In turn, the active $N K D 2$ protein effectively inhibits cell proliferation and colony formation, and it also stops the cell cycle at the G2/M control point. Consecutively, the reactivation of the NKD2 gene expression sensitized the stomach cancer cells to docetaxel. The analysis of the expression level of over 200 genes by using the expression microarray technique indicated the existence of a molecular mechanism of inhibition of the expression of the SOX18 transcription factor via the NKD2 protein. SOX18 expression significantly correlated positively with the methylation profile of the NKD2 gene promoter. It was also shown that SOX18 directly affects the levels of the metalloproteinases MMP-2, MMP-7 and MMP-9 (42). Due to the fact that the methylation of the NKD2 gene promoter entails a reduced expression of the SOX18 factor (which ultimately results in a decrease in the metalloproteinase level), SOX18 was found to play a key role in the progression and metastasis of stomach cancer cells.

In tests on material from 75 cases of primary liver cancer and the hepatocellular carcinoma (HCC) human cell lines HepG2, BEL-7404, SMC-7721, MHCC-97L and MHCC-97H, Wang et al (43) tried to determine the role of the SOX18 transcription factor in the pathogenesis of this cancer. A significantly higher level of SOX18 in cases of liver cancer was observed compared to cases of normal liver tissue from the area surrounding the tumor, both at the mRNA and protein levels. This high level of SOX18 correlated with a shorter DFS period. The in vitro tests carried out in order to inhibit the SOX18 expression clearly showed that a decrease in the level of the SOX18 protein resulted in a cell cycle arrest at the G1 control point and the initiation of apoptosis in liver cancer cells (43). SOX18-siRNA significantly reduced the ability of the HCC cells to migrate and invade. The authors were also able to show the contribution of the SOX18 protein in tumor progression through direct interaction with the proteins Ras homolog family member A (RhoA), insulin-like growth factor 1 receptor (IGF1R), platelet derived growth factor Subunit B (PDGFB), C-C Motif Chemokine Ligand (CCL) 2, CCL3 and CCL5, which are responsible, among others, for focal adhesion and signalling in the chemokine signalling pathway (43). 


\section{Non-small cell lung cancer}

Lung malignant tumors arise as a result of the accumulation of damage to the genetic material of the cells, which ultimately leads to the process of tumor transformation (44).

Numerous studies have shown that in NSCLCs mutations appear most commonly in genes belonging to pathways which are related to, among others, the activation of the epidermal growth factor receptor protein (EGFR), i.e., RAS/RAF/MEK/ERK and PI3K, AKT/mTOR (45). The result of this is an enhanced proliferation of cancer cells and their ability to form metastasis, as well as the intensification of the angiogenesis processes within the tumor. EGFR membrane expression (from the tyrosine kinase family) is relatively frequent in NSCLC cells, in contrast to small-cell lung cancer and unchanged tissue. EGFR overexpression is observed in $40-80 \%$ of NSCLC cases, including $24-89 \%$ of SQCs and $23-46 \%$ of ACs (46).

There are epigenetic mechanisms that are responsible for the gene and protein expression profile specific for a determined tissue and regulate it with precision. The basic epigenetic mechanisms include DNA methylation, post-translational modifications of proteins and miRNA or siRNA participation (47). These mechanisms often seem to have a different course in cancer cells than in normal human cells (48). It has been recently proven that in cases of non-small cell lung cancer (NSCLC) there is a high heterogeneity of the methylation process $(49,50)$. For example, non-malignant lung tissue (NMLT) is characterized by a high level of methylation of the SOX18 gene promoter. In turn, the closer to the tumor area, the lower this level is. Therefore, it should be assumed that also in the tumor itself, depending on the area being studied, the level of methylation varies and can affect the neoplastic process (51). Azhikina et al (51), in lung cancer post-operative material and cases of NMLT, determined the methylation profiles of four gene promoters: Tissue inhibitor of metalloproteinases 4 (TIMP4), GATA binding protein 4 (GATA4), multiple epidermal growth factor-like domains protein 7 (EGFL7) and SOX18, whose role in the neoplastic process was confirmed and documented. They demonstrated the existence of the heterogeneity of the methylation level of the SOX18 gene promoter both in NSCLC and NMLT cases, and pointed out the possibility of using the methylation profile of the SOX18 gene promoter in NSCLC diagnosis in the future (51).

The results so far seem to confirm the complexity of the epigenetic mechanisms in NSCLC $(9,16)$. Based on the results obtained from our research, different SOX18 expressions were observed in cases of NSCLC both at the mRNA and protein levels (Fig. 2) (9,16). Interestingly, the level of mRNA did not in any way reflect the level of protein, determined with the use of the western blot technique (16). Later experiments revealed the participation of two miRNA molecules in this phenomenon: miR-7a and miR-24-3p (9). The results of the analysis of the level of these molecules in cases of lung adenocarcinoma (AC) and squamous-cell lung carcinoma (SQC) and their corresponding cases of NMLT point to a mechanism carried out by the cancer cells through which they reduce the primary high level of the miRNA molecules found in normal lung tissue $(9,52)$. Therefore, the demonstration of the role of specific miRNAs and the role of the methylation of the SOX18 gene promoter in the above-described mechanism may be used in the future in diagnostics, prognostic assessment and, possibly, in NSCLC targeted therapy as well.

The prospect of using miRNA interacting with the SOX18 gene in the treatment of non-small cell lung carcinomas seems to be promising, since there are already many similar miRNA molecules with proven pro- or anti-cancer functions $(53,54)$. Variable miRNA molecule levels can be detected in the patient's blood, which further increases the availability and universality of their use in lung cancer diagnosis. Besides, miRNA examination can provide information not only about the type, but also about the stage of the cancer.

\section{Skin lymphomas}

Jankowska et al (55) attempted to analyse the level of expression of the SOX18 transcription factor in 80 cases of mycosis fungoides (MF). A positive correlation was found between the increase in the SOX18 level in MF cases and the progression of the neoplastic disease. In contrast to previous publications in which SOX18 expression was observed both in tumor cells and in the nuclei of vascular endothelial cells, the results in these studies indicated the presence of SOX18 expression only in the vascular endothelial cells (54). Hence, the authors postulate that in this type of cancer the role of SOX18 is limited to the process of angiogenesis itself, which is key in cancer progression, allowing the tumor to nourish, grow and metastasize. This observation is supported by the fact that a statistically higher level of SOX18 was observed in the blood vessels in the cases of MF compared to neoplasms like lichen planus and eczema. In addition, the high levels of SOX18 correlated positively with the degree of histological differentiation of the MF, which confirms the role of this transcription factor in the process of tumor progression (55). The differences between the mRNA and the SOX18 protein levels in MF cases may indicate, as it happens in other types of cancers, the complexity of the modulation process of the transcript of this gene through the participation of, for example, epigenetic factors. The observation of the existence of a positive correlation between the SOX18 expression and the expression of known proliferation markers such as antigen identified by monoclonal antibody Ki-67 (Ki-67), Minichromosome Maintenance Complex (MCM) $-3,-7$, suggests that the SOX18 protein could be used in the future as a potential marker of tumor aggressiveness.

\section{Osteosarcoma}

An increased expression of the SOX18 transcription factor was observed in osteosarcoma cells, a fact that may be of great importance in the future in the diagnosis and therapy of this type of malignant tumor (56). In the in vitro and in vivo tests proposed by Wu et al, the SOX18 expression was effectively silenced in the human osteosarcoma cell lines U2OS and MG63, which resulted in a decrease in the cancer cells' ability to form metastases and inhibited tumor growth, thus confirming the role of SOX18 in tumor cell proliferation. Moreover, it was noted that the inhibition of the SOX18 expression results in a cell cycle arrest in the $\mathrm{S}$ phase, and thus results in tumor cell apoptosis (56). Furthermore, there was a reduction of the osteosarcoma cell adhesion and migration capacity. The mechanism that underlies 
the observed phenomena is still unclear, although the authors hypothesize that SOX18 could be involved in the pathway of the activation of the proteins platelet-derived growth factor $A$ (PDGFA), PDGFB, RhoA and transforming growth factor- $\beta 1$ (TGF- $\beta 1$ ), which are responsible for cancer cell progression and invasion. As previously described, a similar mechanism was also observed in liver cancer cells (43). The results of the studies presented above seem to attribute a suppressor role to the SOX18 transcription factor in osteosarcoma cells.

\section{Conclusions}

It has been found that the SOX18 protein may be expressed differently in some types of human malignant cancers, although the mechanisms underlying the biological basis of this process are still not fully understood. The SOX proteins are involved in the same pathways as the VEGFs, which indicates their participation in the development of the cardiovascular system and in lymphangiogenesis (28). The proteins from this group exhibit a fairly high homology, and therefore they are able to take over each other's functions in specific situations. It has been demonstrated, in the absence of the SOX18 protein, its function, which is the development of the lymphatic vessels in mice, its fulfilled by the SOX7 and SOX17 proteins (57).

An in-depth study of the epigenetic mechanisms conditioning these phenomena may allow a more comprehensive understanding of the molecular basis of cancer development, which in turn may contribute to the implementation of new targeted therapy based ultimately on proteins from the SOX family (a representative of which is SOX18). Great efforts have been recently made in order to fully understand the multifactorial and multistage process of carcinogenesis. Since epigenetic changes are also at the basis of the cancer process, the discovery of new markers of susceptibility to cancer based, among others, on miRNA-based regulation can enable the introduction into clinical practice of new diagnostic and predictive tests, and perhaps also of molecular therapies.

Considering the role of the proteins of the SOX family in the progression of many types of human malignant tumors and their role in the proliferation and migration processes and in cell apoptosis, it is reasonable to test, identify and understand the mechanisms in which the SOX transcription factors (including the SOX18 protein) play a key role.

\section{Acknowledgements}

Not applicable.

\section{Funding}

No funding was received.

\section{Availability of data and materials}

Not applicable.

\section{Authors' contributions}

MO was involved in the preparation of the manuscript, gathering of the necessary publications and figure preparation.
PD and MPO contributed to revising the manuscript and language.

\section{Ethics approval and consent to participate}

Not applicable.

\section{Consent for publication}

Not applicable.

\section{Competing interests}

The authors declare that they have no competing interests.

\section{References}

1. Gubbay J, Collignon J, Koopman P, Capel B, Economou A, Münsterberg A, Vivian N, Goodfellow P and Lovell-Badge R: A gene mapping to the sex-determining region of the mouse $\mathrm{Y}$ chromosome is a member of a novel family of embryonically expressed genes. Nature 346: 245-250, 1990.

2. Harley VR, Lovell-Badge R and Goodfellow PN: Definition of a consensus DNA binding site for SRY. Nucleic Acids Res 22: 1500-1501, 1994

3. Lovell-Badge R: The early history of the Sox genes. Int J Biochem Cell Biol 42: 378-380, 2010.

4. Wegner M: From head to toes: The multiple facets of Sox proteins. Nucleic Acids Res 27: 1409-1420, 1999.

5. Bowles J, Schepers G and Koopman P: Phylogeny of the SOX family of developmental transcription factors based on sequence and structural indicators. Dev Biol 227: 239-255, 2000.

6. Taniguchi K, Hiraoka Y, Ogawa M, Sakai Y, Kido S and Aiso S: Isolation and characterization of a mouse SRY-related cDNA, mSox7. Biochim Biophys Acta 1445: 225-231, 1999.

7. Kanai Y, Kanai-Azuma M, Noce T, Saido TC, Shiroishi T, Hayashi Y and Yazaki K: Identification of two Sox17 messenger RNA isoforms, with and without the high mobility group box region, and their differential expression in mouse spermatogenesis. J Cell Biol 133: 667-681, 1996.

8. Dunn TL, Mynett-Johnson L, Wright EM, Hosking BM Koopman PA and Muscat GE: Sequence and expression of Sox-18 encoding a new HMG-box transcription factor. Gene 161: 223-225, 1995.

9. Olbromski M, Grzegrzolka J, Jankowska-Konsur A Witkiewicz W, Podhorska-Okolow $M$ and Dziegiel P: MicroRNAs modulate the expression of the SOX18 transcript in lung squamous cell carcinoma. Oncol Rep 36: 2884-2892, 2016.

10. Irrthum A, Devriendt K, Chitayat D, Matthijs G, Glade C, Steijlen PM, Fryns JP, Van Steensel MA and Vikkula M: Mutations in the transcription factor gene SOX18 underlie recessive and dominant forms of hypotrichosis-lymphedema-telangiectasia. Am J Hum Genet 72: 1470-1478, 2003.

11. Cermenati S, Moleri S, Cimbro S, Corti P, Del Giacco L, Amodeo R, Dejana E, Koopman P, Cotelli F and Beltrame M: Sox18 and Sox7 play redundant roles in vascular development. Blood 111: 2657-2666, 2008.

12. Castillo SD and Sanchez-Cespedes M: The SOX family of genes in cancer development: Biological relevance and opportunities for therapy. Expert Opin Ther Targets 16: 903-919, 2012.

13. Pula B, Werynska B, Olbromski M, Muszczynska-Bernhard B, Chabowski M, Janczak D, Zabel M, Podhorska-Okolow M and Dziegiel P: Expression of Nogo isoforms and Nogo-B receptor (NgBR) in non-small cell lung carcinomas. Anticancer Res 34: 4059-4068, 2014.

14. Pula B, Olbromski M, Wojnar A, Gomulkiewicz A, Witkiewicz W, Ugorski M, Dziegiel P and Podhorska-Okolow M: Impact of SOX18 expression in cancer cells and vessels on the outcome of invasive ductal breast carcinoma. Cell Oncol (Dordr) 36: 469-483, 2013.

15. Pula B, Kobierzycki C, Solinski D, Olbromski M, Nowak-Markwitz E, Spaczynski M, Kedzia W, Zabel M and Dziegiel P: SOX18 expression predicts response to platinum-based chemotherapy in ovarian cancer. Anticancer Res 34: 4029-4037, 2014. 
16. Jethon A, Pula B, Olbromski M, Werynska B Muszczynska-Bernhard B, Witkiewicz W, Dziegiel P and Podhorska-Okolow M: Prognostic significance of SOX18 expression in non-small cell lung cancer. Int J Oncol 46: 123-132, 2015.

17. Zhu Y, Li Y, Jun Wei JW and Liu X: The role of Sox genes in lung morphogenesis and cancer. Int J Mol Sci 13: 15767-15783, 2012.

18. Sekido R: A transcriptional activator of mammalian testis determination. Int J Biochem Cell Biol 42: 417-420, 2010.

19. Medina PP, Castillo SD, Blanco S, Sanz-Garcia M, Largo C, Alvarez S, Yokota J, Gonzalez-Neira A, Benitez J, Clevers HC, et al: The SRY-HMG box gene, SOX4, is a target of gene amplification at chromosome $6 \mathrm{p}$ in lung cancer. Hum Mol Genet 18: 1343-1352, 2009.

20. Penzo-Méndez AI: Critical roles for SoxC transcription factors in development and cancer. Int J Biochem Cell Biol 42: 425-428, 2010.

21. Lefebvre V: The SoxD transcription factors-Sox5, Sox6, and Sox13-are key cell fate modulators. Int J Biochem Cell Biol 42: 429-432, 2010

22. Haldin CE and LaBonne C: SoxE factors as multifunctional neural crest regulatory factors. Int J Biochem Cell Biol 42: 441-444, 2010.

23. Stolt CC and Wegner M: SoxE function in vertebrate nervous system development. Int J Biochem Cell Biol 42: 437-440, 2010.

24. Barrionuevo F and Scherer G: SOX E genes: SOX9 and SOX8 in mammalian testis development. Int J Biochem Cell Biol 42: 433-436, 2010

25. Ito M: Function and molecular evolution of mammalian Sox 15 , a singleton in the SoxG group of transcription factors. Int $\mathrm{J}$ Biochem Cell Biol 42: 449-452, 2010

26. Han F, Wang Z, Wu F, Liu Z, Huang B and Wang D: Characterization, phylogeny, alternative splicing and expression of Sox30 gene. BMC Mol Biol 11: 98, 2010.

27. Han F, Liu W, Jiang X, Shi X, Yin L, Ao L, Cui Z, Li Y, Huang C, Cao J and Liu J: SOX30, a novel epigenetic silenced tumor suppressor, promotes tumor cell apoptosis by transcriptional activating p53 in lung cancer. Oncogene 34: 4391-4402, 2015.

28. François M, Caprini A, Hosking B, Orsenigo F, Wilhelm D, Browne C, Paavonen K, Karnezis T, Shayan R, Downes M, et al: Sox18 induces development of the lymphatic vasculature in mice. Nature 456: 643-647, 2008

29. Sakamoto Y, Hara K, Kanai-Azuma M, Matsui T, Miura Y, Tsunekawa N, Kurohmaru M, Saijoh Y, Koopman P and Kanai Y: Redundant roles of Sox17 and Sox18 in early cardiovascular development of mouse embryos. Biochem Biophys Res Commun 360: 539-544, 2007.

30. Bernard P and Harley VR: Acquisition of SOX transcription factor specificity through protein-protein interaction, modulation of Wnt signalling and post-translational modification. Int J Biochem Cell Biol 42: 400-410, 2010.

31. Hosking BM, Wang SC, Chen SL, Penning S, Koopman P and Muscat GE: SOX18 directly interacts with MEF2C in endothelial cells. Biochem Biophys Res Commun 287: 493-500, 2001.

32. Sinner D, Rankin S, Lee M and Zorn AM: Sox 17 and beta-catenin cooperate to regulate the transcription of endodermal genes. Development 131: 3069-3080, 2004.

33. Kormish JD, Sinner D and Zorn AM: Interactions between SOX factors and Wnt/beta-catenin signaling in development and disease. Dev Dyn 239: 56-68, 2010.

34. Sinner D, Kordich JJ, Spence JR, Opoka R, Rankin S, Lin SC Jonatan D, Zorn AM and Wells JM: Sox 17 and Sox4 differentially regulate beta-catenin/T-cell factor activity and proliferation of colon carcinoma cells. Mol Cell Biol 27: 7802-7815, 2007.

35. Chan DW, Mak CS, Leung TH, Chan KK and Ngan HY: Down-regulation of Sox7 is associated with aberrant activation of Wnt/b-catenin signaling in endometrial cancer. Oncotarget 3 : 1546-1556, 2012.

36. Saitoh T and Katoh M: Expression of human SOX18 in normal tissues and tumors. Int J Mol Med 10: 339-344, 2002.

37. García-Ramírez M, Martínez-González J, Juan-Babot JO, Rodríguez C and Badimon L: Transcription factor SOX18 is expressed in human coronary atherosclerotic lesions and regulates DNA synthesis and vascular cell growth. Arterioscler Thromb Vasc Biol 25: 2398-2403, 2005.

38. Darby IA, Bisucci T, Raghoenath S, Olsson J, Muscat GE and Koopman P: Sox18 is transiently expressed during angiogenesis in granulation tissue of skin wounds with an identical expression pattern to Flk-1 mRNA. Lab Invest 81: 937-943, 2001.

39. Kim I and Koh GY: Taking aim at Sox18. Elife 6: pii: e24238, 2017.
40. Young N, Hahn CN, Poh A, Dong C, Wilhelm D, Olsson J, Muscat GE, Parsons P, Gamble JR and Koopman P: Effect of disrupted SOX18 transcription factor function on tumor growth, vascularization, and endothelial development. J Natl Cancer Inst 98: 1060-1067, 2006.

41. Petrovic I, Milivojevic M, Popovic J, Schwirtlich M, Rankovic B and Stevanovic M: SOX18 is a novel target gene of hedgehog signaling in cervical carcinoma cell lines. PLoS One 10: e0143591, 2015.

42. Jia Y, Cao B, Yang Y,LinghuE,Zhan Q, Lu Y, Yu Y,Herman JG and Guo M: Silencing NKD2 by promoter region hypermethylation promotes gastric cancer invasion and metastasis by up-regulating SOX18 in human gastric cancer. Oncotarget 6: 33470-33485, 2015.

43. Wang G, Wei Z, Jia H, Zhao W, Yang G and Zhao H: Knockdown of SOX18 inhibits the proliferation, migration and invasion of hepatocellular carcinoma cells. Oncol Rep 34: 1121-1128, 2015.

44. Hanahan D and Weinberg RA: Hallmarks of cancer: The next generation. Cell 144: 646-674, 2011.

45. Ma L, Liu L, Zhang T and Shan L: Detection and evaluation of EGFR mutation status in serum of patients with advanced non-small cell lung cancer treated with EGFR-TKIs. Zhongguo Fei Ai Za Zhi 16: 303-307, 2013 (In Chinese).

46. Meert AP, Martin B, Delmotte P, Berghmans T, Lafitte JJ, Mascaux C, Paesmans M, Steels E, Verdebout JM and Sculier JP: The role of EGF-R expression on patient survival in lung cancer: A systematic review with meta-analysis. Eur Respir J 20: 975-981, 2002.

47. Friedman RC, Farh KK, Burge CB and Bartel DP: Most mammalian mRNAs are conserved targets of microRNAs. Genome Res 19: 92-105, 2009.

48. Holzner B, Kemmler G, Greil R, Kopp M, Zeimet A, Raderer M, Hejna M, Zöchbauer S, Krajnik G, Huber H, et al: The impact of hemoglobin levels on fatigue and quality of life in cancer patients. Ann Oncol 13: 965-973, 2002.

49. Guo SC, Yan F, Xu J, Bao Y, Zhu J, Wang X, Wu J, Li Y, Pu W, Liu $\mathrm{Y}$, et al: Identification and validation of the methylation biomarkers of non-small cell lung cancer (NSCLC). Clin Epigenetics 7: 3, 2015.

50. Huang T, Chen X, Hong Q, Deng Z, Ma H, Xin Y, Fang Y, Ye H, Wang R, Zhang C, et al: Meta-analyses of gene methylation and smoking behavior in non-small cell lung cancer patients. Sci Rep 5: 8897, 2015.

51. Azhikina T, Kozlova A, Skvortsov $\mathrm{T}$ and Sverdlov E: Heterogeneity and degree of TIMP4, GATA4, SOX18, and EGFL7 gene promoter methylation in non-small cell lung cancer and surrounding tissues. Cancer Genet 204: 492-500, 2011.

52. Olbromski M, Rzechonek A, Grzegrzolka J, Glatzel-Plucinska N, Chachaj A, Werynska B, Podhorska-Okolow M and Dziegiel P: Influence of miR-7a and miR-24-3p on the SOX18 transcript in lung adenocarcinoma. Oncol Rep 39: 201-208, 2018.

53. Zhang C, Ge S, Hu C, Yang N and Zhang J: MiRNA-218, a new regulator of HMGB1, suppresses cell migration and invasion in non-small cell lung cancer. Acta Biochim Biophys Sin (Shanghai) 45: 1055-1061, 2013.

54. Xie C,Han Y,Liu Y,Han L and Liu J: miRNA-124 down-regulates SOX8 expression and suppresses cell proliferation in non-small cell lung cancer. Int J Clin Exp Pathol 7: 7518-7526, 2014.

55. Jankowska-Konsur A, Kobierzycki C, Reich A, Piotrowska A, Gomulkiewicz A, Olbromski M, Podhorska-Okołów M, Dzięgiel P and Szepietowski JC: Expression of SOX18 in mycosis fungoides. Acta Derm Venereol 97: 17-23, 2017.

56. Wu Z, Liu J, Wang J and Zhang F: SOX18 knockdown suppresses the proliferation and metastasis, and induces the apoptosis of osteosarcoma cells. Mol Med Rep 13: 497-504, 2016.

57. Bernard P and Harley VR: Acquisition of SOX transcription factor specificity through protein-protein interaction, modulation of Wnt signalling and post-translational modification. Int J Biochem Cell Biol 42: 400-410, 2010.

58. Image from the RCSB PDB of 4Y60: Structure and decoy-mediated inhibition of the SOX18/Prox1-DNA interaction. Klaus M., Prokoph N., Girbig M., Wang X., Huang Y.H., Srivastaya Y., Hou L., Narasimhan K., Kolatkar P.R., Francois M., Jauch R. (2016) Nucleic Acids Res. 44: 3922-3935. 